\title{
The Nerve to Give BACE Inhibitors a Second Chance?
}

\author{
Robert H. Baloh ${ }^{1}$
}

Published online: 8 June 2020

(C) The American Society for Experimental NeuroTherapeutics, Inc. 2020
Inhibitors of $\beta$-site amyloid precursor protein (APP)-cleaving enzyme (BACE) were once a major focus of the pharmaceutical industry because of their prospects for treating Alzheimer's disease (AD) [1]. Following the discovery of BACE1 in 1999 [2-5] as an aspartyl protease that cleaves APP to generate aggregation-prone fragments, the potential to develop BACE inhibitors to treat $\mathrm{AD}$ was immediately apparent, and many pharmacologic inhibitors were engineered [6]. However, after 20 years and repeated failures in costly late-stage pivotal trials, BACE inhibition as a therapeutic strategy for $A D$ is all but abandoned [7]. Among the problems for BACE inhibitors appears to be that BACE1 is a promiscuous protease, with over 60 substrates now recognized [8]. This promiscuity leads to significant potential for unwanted side effects due to altered cleavage of proteins other than APP, several of which regulate synaptic function and may have been responsible for the unexpected worsening of cognition seen in BACE1 inhibitor AD trials [9]. However, in theory, the existence of additional BACE1 substrates provides opportunity to repurpose BACE inhibitors for diseases other than $\mathrm{AD}$.

Not long after its discovery as an APP protease, BACE1 was also found to cleave type III neuregulin 1 , which is critical for axonal-Schwann cell communication during peripheral nerve development, and BACE1 knockout mice demonstrate developmental phenotypes of hypomyelination and abnormal axonal sorting in peripheral nerves $[10,11]$. In addition, BACE1-mediated cleavage of APP was reported to induce axonal degeneration via a death receptor 6 (DR6) and caspase 6 dependent mechanism [12], suggesting multiple roles for BACE1 in mediating peripheral nerve development and degeneration.

Therefore, in 2011, the group led by Drs. Farah and Griffin investigated whether lack of BACE1 altered axonal

Robert H. Baloh

robert.baloh@csmc.edu

1 Department of Neurology, Center for Neural Science and Medicine, Board of Governors Regenerative Medicine Institute, Cedars-Sinai Medical Center, 8700 Beverly Blvd, Los Angeles, CA 90048, USA degeneration and found that while BACE1 knockout axons degenerated normally after axotomy or chemically induced neuropathy, they unexpectedly found accelerated axonal regeneration and enhanced reinnervation of neuromuscular junctions [13]. Additionally, they later showed that overexpression of BACE1 inhibited peripheral nerve regeneration [14]. This led to the proposal that a subset of BACE1 substrates in neurons, macrophages, and Schwann cells have a net negative effect on peripheral axon regeneration, and suggested that BACE1 inhibition could be therapeutically useful to treat nerve injury by promoting a growth permissive microenvironment.

In the current issue of Neurotherapeutics, Tallon et al. build on their previous work and demonstrate that pharmacologic BACE inhibition can improve axonal regeneration in an axonal crush model, and enhance terminal sprouting in a partial nerve lesion model [15]. They chose a transient dosing paradigm to address the issue of decreased remyelination predicted from blocking type III neuregulin signaling. While peripheral axons have the capacity to regenerate, the process is generally incomplete and inefficient, and target tissues undergo atrophy and death, which precludes functional recovery $[16,17]$. Therefore, agents that improve regeneration after peripheral nerve injury are an area of unmet medical need.

In addition to a peripheral nerve injury model, Tallon et al. treated $\mathrm{SOD} 1^{\mathrm{G} 93 \mathrm{~A}}$ transgenic mice, a model of amyotrophic lateral sclerosis (ALS), with a BACE1 inhibitor and observed that while there was transient benefit in functional innervation, this effect disappeared over time. This finding mirrors the results of other attempts at using axonal protective therapies in preclinical models of motor neuron disease [18-20], where axonal protective therapies provided only transient benefit early in disease. This leaves the prospects of BACE1 inhibitors in ALS less certain, since it is unclear whether transient axonal protection would have clinical benefit for patients with the disease.

While the authors were able to show that cleavage of the BACE1 substrate Close Homolog of L1 (CHL1) is diminished in $\mathrm{SOD} 1^{\mathrm{G} 93 \mathrm{~A}}$ transgenic mice, a key question remains unanswered - what is/are the substrate(s) that mediate the 
promotion of a growth permissive environment by BACE1 inhibition in peripheral nerves? A better understanding of the mechanism could lead to development of more substrate focused therapeutic agents to improve peripheral nerve regeneration. Regardless, significant challenges remain in further advancement toward clinical application for peripheral nerve diseases. The variability of injury mechanism and recovery after peripheral nerve trauma in general makes it difficult to design a trial to properly demonstrate clinical benefit of axonal protective agents [21]. Likewise, long-term treatment may be problematic due to unwanted side effects from inhibition of a variety of BACE1 substrates in both the central and peripheral nervous system, limiting application for chronic acquired or inherited peripheral neuropathies. However, several of these (e.g., chemotherapy-induced neuropathy, Charcot-MarieTooth disease, etc.) have a more tractable path in terms of natural history data and outcomes measures for clinical trial design, and if $\mathrm{BACE}$ inhibition can find the right balance to generate a growth permissive environment for peripheral axons, then perhaps someone will have the nerve to give BACE1 inhibitors a second chance.

Required Author Forms Disclosure forms provided by the authors are available with the online version of this article.

\section{References}

1. Yan, R. and R. Vassar, Targeting the beta secretase BACE1 for Alzheimer's disease therapy. Lancet Neurol, 2014. 13(3): p. 319-29.

2. Sinha, S., et al., Purification and cloning of amyloid precursor protein beta-secretase from human brain. Nature, 1999. 402(6761): p. 537-40.

3. Hussain, I., et al., Identification of a novel aspartic protease (Asp 2) as beta-secretase. Mol Cell Neurosci, 1999. 14(6): p. 419-27.

4. Vassar, R., et al., Beta-secretase cleavage of Alzheimer's amyloid precursor protein by the transmembrane aspartic protease BACE. Science, 1999. 286(5440): p. 735-41.

5. Yan, R., et al., Membrane-anchored aspartyl protease with Alzheimer's disease beta-secretase activity. Nature, 1999. 402(6761): p. 533-7.
6. Silvestri, R., Boom in the development of non-peptidic betasecretase (BACE1) inhibitors for the treatment of Alzheimer's disease. Med Res Rev, 2009. 29(2): p. 295-338.

7. Mullard, A., BACE failures lower AD expectations, again. Nat Rev Drug Discov, 2018. 17(6): p. 385.

8. Hemming, M.L., et al., Identification of beta-secretase (BACE1) substrates using quantitative proteomics. PLoS One, 2009. 4(12): p. e8477.

9. Egan, M.F., et al., Randomized Trial of Verubecestat for Prodromal Alzheimer's Disease. N Engl J Med, 2019. 380(15): p. 1408-1420.

10. $\mathrm{Hu}, \mathrm{X}$., et al., Bacel modulates myelination in the central and peripheral nervous system. Nat Neurosci, 2006. 9(12): p. 1520-5.

11. Willem, M., et al., Control of peripheral nerve myelination by the beta-secretase BACE1. Science, 2006. 314(5799): p. 664-6.

12. Nikolaev, A., et al., APP binds DR6 to trigger axon pruning and neuron death via distinct caspases. Nature, 2009. 457(7232): p. 981-9.

13. Farah, M.H., et al., Reduced BACE1 activity enhances clearance of myelin debris and regeneration of axons in the injured peripheral nervous system. J Neurosci, 2011. 31(15): p. 5744-54.

14. Tallon, C., et al., Increased BACE1 activity inhibits peripheral nerve regeneration after injury. Neurobiol Dis, 2017. 106: p. 147157.

15. Tallon, C., et al., Pharmacological BACE Inhibition Improves Axonal Regeneration in Nerve Injury and Disease Models. Neurotherapeutics, 2020.

16. Gordon, T., et al., Accelerating axon growth to overcome limitations in functional recovery after peripheral nerve injury. Neurosurgery, 2009. 65(4 Suppl): p. A132-44.

17. Hoke, A., Mechanisms of Disease: what factors limit the success of peripheral nerve regeneration in humans? Nat Clin Pract Neurol, 2006. 2(8): p. 448-54.

18. Vande Velde, C., et al., The neuroprotective factor Wlds does not attenuate mutant SOD1-mediated motor neuron disease. Neuromolecular Med, 2004. 5(3): p. 193-203.

19. Wang, L., et al., Mitofusin 2 Regulates Axonal Transport of Calpastatin to Prevent Neuromuscular Synaptic Elimination in Skeletal Muscles. Cell Metab, 2018. 28(3): p. 400-414 e8.

20. Peters, O.M., et al., Loss of Sarm1 does not suppress motor neuron degeneration in the SOD1G93A mouse model of amyotrophic lateral sclerosis. Hum Mol Genet, 2018. 27(21): p. 3761-3771.

21. Scheib, J. and A. Hoke, Advances in peripheral nerve regeneration. Nat Rev Neurol, 2013. 9(12): p. 668-76.

Publisher's Note Springer Nature remains neutral with regard to jurisdictional claims in published maps and institutional affiliations. 